\title{
Metacognitive Scaffolding Agent Based on BDI Model for Interactive Learning Environments
}

\author{
Anass Hsissi, Hakim Allali, and Abdelmajid Hajami
}

\begin{abstract}
This article is based on doctoral research in progress whose main objective is the integration of metacognitive dimension in the design of interactive learning environments. We conducted a theoretical analysis with the aim of defining the pedagogical functions must ensure that our ILE so it can provide scaffolding for metacognitive learners. A literature review has allowed us to define the structure of an activity of self-regulated learning and the ways in which metacognitive scaffolding can be integrated into an activity supported by an ILE. Thus, through this work we propose the model of self-regulated learning activity integrating metacognitive scaffolding based on BDI, a theory for describing behavior of individual.
\end{abstract}

Index Terms-Metacognition, scaffolding, BDI, EIAH, self-regulated.

\section{INTRODUCTION}

Learning is a process wherewith we make the student aware and responsible towards the construction of his knowledge and its use in various contexts and situations [1]. This process is facilitated, supported and arranged by a parallel pedagogical approach of the tutor.

Within the context of EIAH, this learning is favored, raised, supported and validated by intelligent programs. The learner is usually alone in from of the machine; he puts himself in a learning situation. If he can't delimit his objectives, select and undertake the appropriate activities to achieve them, evaluate his own progress, identify the sources of his potential difficulties, take the appropriate decisions, adjust and regulate his steps, many problems will come out while learning. Therefore, the learner could give up using EIAH. Only the learners who were able to develop the steps fore-mentioned before can benefit from the computer tool. The autonomy of the apprentice is related to the concept of self-regulated learning (Butler and Winnie, 1995).

In another context, the metacognitive skills which are an important component of the cognitive development of the learner are unfortunately not explicitly incorporated into learning processes that are transmitted by the programs. They develop implicitly and indirectly as a result of other learning processes and experiences, which does not help the majority of the learners in developing this potential. [Pintrich, McKeachie, and Lin, 1987] [Hofer, Yu, and Pintrich, 1998].

EIAHs don't take into consideration this metacognitive dimension, they don't considerate the learner beyond his

Manuscript received December 3, 2013; revised January 10, 2014.

The aurhors are with the Hassan 1st University, Faculty of Sciences and Technologies LAVETE laboratory, Morocco (e-mail: Anasshsissi@gmail.com, hakim-allali@hotmail.fr). cognitive activity related to the mediatized content. EIAHs that aim to make a metacognitive scaffolding during the learning are rare. [Romero, M., 2005].

The objective of this doctoral research is to find out how to integrate the metacognitive dimension within the learning activity through a EIAH, in the form of a scaffolding in order to stimulate the activation of metacognitive strategies of the learner [Thillmann, Künsting,Wirth et Leutner, 2009]who finds it difficult to use them.

\section{MetaCognition In Learning Situation}

\section{A. The Learning Situation}

From a didactic point of view, the mediatized learning activity in an intelligent environment involves 3 different stages [2]:

Stimulation: the moment when the intelligent system presents the situational problem for the first time to the learner. At this moment, the learner is placed in contact which should put him in a cognitive imbalance when he discovers that his representations are insufficient to resolve the problem.

The construction of new knowledge: the moment when a series of changes of internal structures are established in the actions of the learner. At this moment, the learner regulates himself in terms of metacognition, in order to adapt his learning strategies.

The evaluation phase: Moment of setting a situation problem similar to the one set on the first stage. It is necessary for controlling the learning process, and conceiving remedial pedagogic devices if any significant errors are detected.

We propose in our doctoral research a model of a learning activity that can serve as a framework for the IT development of an EIAH.

This model involves 5 steps:

\begin{tabular}{|l|l|}
\hline $\begin{array}{l}\text { Metacognitive verbalization of emotions related } \\
\text { to the nature of the problem occurs just after the } \\
\text { moment of stimulation [3] }\end{array}$ & The seed phase \\
\hline Preparation, performance, evaluation & $\begin{array}{l}3 \text { phases to cover } \\
\text { construction } \\
\text { moments }\end{array}$ \\
\hline Moment of metacognitive reflection & The distancing phase \\
\hline $\begin{array}{l}\text { prompt, Monitoring prompt, and evaluation } \\
\text { prompt }\end{array}$ & $\begin{array}{l}\text { metacognents of } \\
\text { provocation }\end{array}$ \\
\hline $\begin{array}{l}\text { Distancing verbalization by comparing: The } \\
\text { EIAH must provide a functionality to keep track } \\
\text { of the process adopted by the learner to solve the } \\
\text { problem }\end{array}$ & $\begin{array}{l}\text { 2 moments of } \\
\text { metacognitive } \\
\text { verbalization }\end{array}$ \\
\hline
\end{tabular}




\section{B. Metacognition: A Key to Success}

One of the best indicators of learning success is the ability of the students to reflect on their knowledge and understanding of the reasononing they engage in using and constructing new knowledge. We must therefore make students aware of the learning strategies they implement to learn and understand and achieve success in learning situations. Metacognition is inseparable from self-knowledge and self-confidence [4].

Metacognition consists of having a mental activity [5], [6] of one's own mental processes, i.e. "think on one's own thoughts." Shedding metacognitive light on the learning process of the learner is a complex act of thought, an act of internalization; the learner begins to consciously or unconsciously hold a dialogue on the task it performs. metacognition does not necessarily require a conscious analysis, because some metacognitive operations could take place unconsciously, not always being a conscious introspection.

There are three basic components of the human memory system: the executor, permanent memory and the working memory (Klatzy 1980), working memory is synonymous with conscious attention, we use it to hold information that we are attending, this element has certain limitations such as limited storage, limited pool of effort, limited time processing. Permanent memory contains all information that has ever been processed, the declarative knowledge and procedural knowledge (how to govern the use of information). The third component of the human memory system is the executor which orchestrates the processing of information between working memory and permanent memory. The executor is assisted via the notion of metacognition (i.e., the capability to understand what needs to be done in a given circumstance).

\section{Metacognitive Agent Model}

We present here our agent architecture based on theories for describing behavior of individuals. The most influential theories with respect to agent technology, it's the BDI paradigm. The agent use this model to allow metacognitive scaffolding and support mentioned in the previous section, the treatment of internal metacognitive reasoning of the learners during a cognitive activity, to promote their abilities to think and promote autonomy [7].

Several architectures from different disciplines like psychology, philosophy and biology can be utilized to build agents with cognitive capabilities.

The BDI model was conceived by Bratman as a theory of human practical reasoning. It reduces the explanation framework for complex human behavior to the motivational stance. This means that the causes for actions are always related to the human desires ignoring other facets of human recognition such as emotions. The strength of the BDI model is the consistent usage of folk psychological notions that closely correspond to the way humans talk about behavioral aspects. The architecture of our agent is composed of four components:

Plans: the strategic vision of the metacognitive agent. The plans identify the strategies that the agent can track during the course of a learning session [8], [9]. A plan consists of three elements: Set of sequences of actions: defines the actions that should be applied, considering the chosen plan to achieve the goal (desires) of the Agent, and to help the users in need. Rules of the execution of the actions: defines the conditions for the different actions, when and how to interact with the user. Rules for updating the plan: defines the conditions and consequences of an update of the plan (and the suppression of the plan of the base of the intent).

Desires: the desires of the agent representing the state of the environment, and sometimes itself, the agent would like to see achieved in the learner, they are formalized as a set of criteria that will be used to evaluate the plans.

Beliefs: the beliefs of the agent on the system. They are the information that the agent has on the environment according to the situations and problems, and are used to calculate the values of desires (criteria). But beliefs do not just represent entities in a kind of one-to-one mapping; they provide a domain-dependent abstraction of entities by highlighting important properties while omitting irrelevant details. This introduces a personal world view inside the agent: The way in which the agent perceives and thinks about the world.

Intention: is the plan chosen. The intentions of an agent are the desires that the agent decided to do or actions that he decided to perform to fulfill his desires.

\section{ARCHITECTURE}

Our intelligent agent model paradigm is inspired by the notion of rational agents based in mental attitudes. And specifically, the BDI model (based on the mental processes of Beliefs, Desires, and Intentions), it shows up as a philosophical model for the modeling of the human rational behavior.

Beliefs are related to everything that the agent knows (both related to the environment and to its internal state) and will be stored in the "belief base" of the agent.

When the agent interacts with the user in order to achieve a common task, they have to agree from time to time on what are the beliefs (or the goals). The problem is that sometimes the negotiation step does not rule out all the conflicts. Even in this case, the group has to make a decision on what are its beliefs (goals) in order to carry on. So, in such cases, an aggregation step is needed following the agents wishes.

So, formally, when a decision has to be made about beliefs (goals) of the agent, we can consider this as a two step process. First, a negotiation step allows agents to try to convince the user. Then an aggregation step states what are the common beliefs (goals) of the group.

The agent use a chatter robot as a type of conversational agent designed to simulate an intelligent conversation with the users via auditory or textual methods.

And a trace based system, which allows the agent to parse process and assist users in their complex and dynamic activities.

Plans are composed by a set of instructions which allow the agent to carry out several actions to try achieving the previously set goals [10]. That is, if the current situation (that the agent knows) is not the desired one (i.e., the situation 
specified by the goals) then the agent will execute the plan or plans to achieve this desired situation The relation between goals and plans will be carried out by a reasoning engine, which will decide what plan will be executed to try satisfying a specific goal Desires are the goals. They consist of making the student does a series of educational activities, which have previously defined by a teacher of the platform. Usually these activities will be included in a semantic schema which defines the relations among several learning objects. These relations will make a learning path, which the student must follow. This path, obviously, will not have to be linear, but in our prototype it will have a structure divided into Courses, Modules, Units, and Elements the main objective will be that the student achieves certain kind of competencies related to the taught matter. To do this, this objective will be divided into others sub-objectives do this, this objective will be divided into others sub-objective are achieved, then the high level ones will be achieved too (and so on until achieving the main initial objective).

Intentions are the plans the agent owns to try the student reaches the set out goals. So, the agent will execute a series of plans (actions) taking into account at every time the beliefs which it has, the goals to achieve at this moment, and the messages that can be received from the student. Besides, a series of plans to do routine tasks are included, such as: a task to process messages (both the received ones and the desired to send), tasks to manage the temporization,etc. This representation of the behavior using mental notions has several benefits, such as to avoid the low level abstraction (because only specific goals to achieve and several plans to do that are presented) and easily understanding the autonomous behavior of the agent (even its prediction).

\section{BDI PLATFORMS}

The Jadex project, the solution adopted for our agent is been developed by the Distributed Systems and Information Systems group of the University of Hamburg. Jadex , it's a kind of reasoning engine following the BDI agents model that facilitate the construction of intelligent agents, allowing to use $\mathrm{XML}$ and Java to make these type of agents.

As we mentioned above, Jadex is the solution adopted for our agent. Nowadays, this reasoning engine has several tools which can be grouped in three sets:

- A Java API and a set of predefined functionalities to make agents programming easy

- A platform which allows the execution of these agents (reasoning engine)

- A set of tools to use in execution time which allow managing and monitoring different aspects of these agents

Agents implemented in Jadex are known as Goal Oriented Agents. This paradigm allows including an abstraction level to the definition of the behavior of the agent, and so the developer can specify some goals to be achieved by the agent, without saying how to do it. Now the agent itself has certain degree of freedom to decide the more appropriate form to achieve those goals.

Jadex takes into account the concepts of beliefs, objectives, and plans, which can be created and managed by the agents. As for the BDI model in general, the beliefs will be any kind of Java object, which will be stored in the belief base of each agent; the objectives will allow to describe what an agent must to reach (not including information about how to reach those objectives); at last, plans will be a set of Java instructions which will allow an agent to do several actions to reach the previous described objectives. A reasoned will set the relations among objectives and plans to decide what plan will be executed at each moment.

The Jadex platform offers a complete set of execution tools to allow managing, testing, and debugging agents. The base of these tools is the Jadex Control Center, which acts as the access point for the rest of the tools (plugins).

Finally, Jadex is trying to be compliant with some standards that facilitate the interoperability among systems. For example, we can find one of the standards presents in Jadex in the interchange of messages among agents, which are adapted to the FIPA standard [11]. Besides, Jadex provides certain predefined functionalities through the called "capabilities", which allow to be reused in different agents. Jadex [12] offers us a simple form to develop an agent-based system without loosing the benefits of the intelligent agents paradigm. All of these characteristics of Jadex are enough reasons to choose it for our system.

\section{CONCLUSION}

The main contribution of this paper is the presentation of a Prototype and model of an intelligent learning agent to maintain the metacognitive skills, which includes capabilities related to EIHA, and ITSs.

More specifically, the main task of the tutoring module is to decide at every moment how to help the user to increase his metacognitive skills.

The main future work related to the scaffolding module is to improve its intelligence. The functional prototype developed has a basic intelligence and it is able to carry out the tasks mentioned in this paper for purposes of checking its viability. Nevertheless, it is essential the improvement of its capabilities to deal with a lot of situations which can arise in a real scenario.

\section{REFERENCES}

[1] D. L. Butler and P. H. Winne, "Feedback and self-regulated learning: a theoretical synthesis," Review of Educational Research, vol. 65, pp. 245-281, 1995.

[2] P. R. Pintrich, W. J. McKeachie, S. L. Yu, and B. K. Hofer, "The role of motivation and self-regulated learning in math and science classrooms," 1995.

[3] M. Romero, "10 principes pour la prise en compte du développement métacognitif dans les EIAH," April 2005.

[4] W. J. McMillan, "Your trust is to understand' - how academically successful students learn, teaching in higher Education," vol. 15, no. 1, pp. 1-13, 2010.

[5] J. H. Flavell, "Metacognition and cognitive monitoring," American Psychologist, vol. 34, pp. 906-911, 1979.

[6] D. Wood, J. S. Bruner, and G. Ross, "The role of tutoring in problem solving," Journal of Child Psychology and Psychiatry and Allied Disciplines, vol. 17, pp. 89-100, 1976.

[7] X. D. Lin and J. D. Lehman, "Supporting learning of variable control in a computer based biology environment: Effects of prompting college students to reflect on their own thinking," Journal of Research in Science Teaching, vol. 36, pp. 837-858, 1999. 
[8] C. Gama, "Integrating metacognition instruction in interactive learning environments," Doctoral dissertation, University of Sussex, 2004.

[9] M. Puustinen and L. Pulkkinen, "Models of self-regulated learning: A review," Scandinavian Journal of Educational Research, vol. 45, no. 3, pp. 269-286, 2001.

[10] FIPA standard specifications. [Online]. Available: http://www.fipa.org/repository/standardspecs.html.

[11] L. Braubach. (2009). Jadex, BDI agent system. Distributed systems and information systems group, University of Hamburg. [Online]. Available: http://jadex.informatik.unihamburg.de/xwiki/bin/view/About/Overvie

[12] N. Howden, R. Ronnquist, A. Hodgson, and A. Lucas, "Intelligent agents: summary of an agent infrastructure," in Proc. the 5th International Conference on Autonomous Agents, 2001.

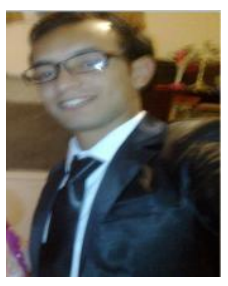

Anass Hsissi was born in 1989 in Morocco, where he still lives and works as a professor of algorithms and programming languages at IT Learning Campus, studied Computer science, his research interest covers several areas, metacognition, ILE: informatics learning environments, eLearning and artificial intelligence. This research involves developing appropriate models and experiments to integrate the metacognitive dimension within the learning activity
Hakim Allali was born in 1966 in Morocco. He received the Ph.D degree from Claude Bernard Lyon I University, France, in 1993 and the "docteur d'etat" degree from Hassan II-Mohamedia University, Casablanca in Morocco in 1997. He is currently a professor at Faculty of Sciences and Technologies of Hassan $1^{\text {st }}$ University of Settat in Morocco and director of LAVETE Laboratory. He is executive manager and founder of IT Learning Campus. His research interests include technology enhanced learning, modeling, image processing and GIS.

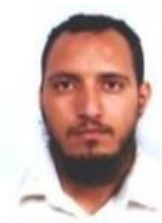

Abdelmajid Hajami was born in Morocco in 1975 . $\mathrm{He}$ received the Ph.D in informatics and telecommunications from Mohamed V-Souissi University Rabat- Morocco. $\mathrm{He}$ is currently an assistant professor at the Faculty of Science and Technology of Settat in Morocco, his research interests include: ILE "informatics Learning Environments", eLearning and Wireless networks security and QoS.

through an EIAH. 